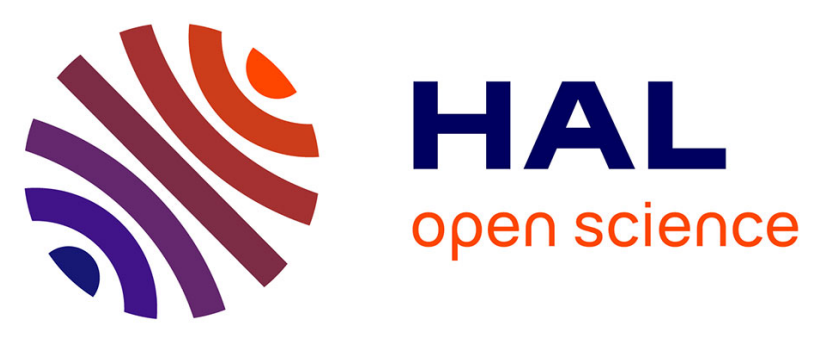

\title{
The segmental progression of early untreated Parkinson disease: a novel approach to clinical rating
}

Michael W M Schüpbach, Jean-Christophe Corvol, Virginie Czernecki, Mouna Ben Djebara, Jean-Louis Golmard, Yves Agid, Andreas Hartmann

\section{- To cite this version:}

Michael W M Schüpbach, Jean-Christophe Corvol, Virginie Czernecki, Mouna Ben Djebara, JeanLouis Golmard, et al.. The segmental progression of early untreated Parkinson disease: a novel approach to clinical rating. Journal of Neurology, Neurosurgery and Psychiatry, 2009, 81 (1), pp.20. 10.1136/jnnp.2008.159699 . hal-00552721

\section{HAL Id: hal-00552721 \\ https://hal.science/hal-00552721}

Submitted on 6 Jan 2011

HAL is a multi-disciplinary open access archive for the deposit and dissemination of scientific research documents, whether they are published or not. The documents may come from teaching and research institutions in France or abroad, or from public or private research centers.
L'archive ouverte pluridisciplinaire HAL, est destinée au dépôt et à la diffusion de documents scientifiques de niveau recherche, publiés ou non, émanant des établissements d'enseignement et de recherche français ou étrangers, des laboratoires publics ou privés. 


\section{The segmental progression of early untreated Parkinson disease: a novel approach to clinical rating}

W. M. Michael Schüpbach, ${ }^{1,2,3,6}$ MD; Jean-Christophe Corvol, ${ }^{1,3,8}$ MD PhD; Virginie

Czernecki, ${ }^{1,3,7}$ PhD; Mouna Ben Djebara, MD ${ }^{1,2,3,4}$; Jean-Louis Golmard, ${ }^{5}$ MD PhD; Yves

Agid, ${ }^{1,2,3,4}$ MD PhD; and Andreas Hartmann, ${ }^{1,2,3,4}$ MD

(1) Centre d'Investigation Clinique, Fédération des Maladies du Système Nerveux, Paris, France

(2) INSERM, UMR 679, Neurology and Experimental Therapeutics, Paris, France

(3) AP-HP, Pitié-Salpêtrière Group, Fédération de Neurologie, Paris, France

(4) Université Pierre et Marie Curie, Faculté de Médecine, Paris, France

(5) AP-HP, Pitié-Salpêtrière Group, Biostatistics Unit, Paris, France

(6) Department of Neurology, Bern University Hospital and University of Bern, Switzerland

(7) INSERM UMR 610, Functional neuroanatomy of normal and pathological behaviour, Paris, France

(8) Service de Pharmacologie, Pitié-Salpêtrière Group, Paris, France

\section{Address for correspondence:}

Andreas Hartmann, MD, Centre d'Investigation Clinique, Pitié-Salpêtrière Group,

47, Boulevard de 1’Hôpital, F - 75651 Paris Cedex 13, France

Tel.: +33142161950

Fax: + 33142161958

E-mail: andreas.hartmann@psl.aphp.fr 
Running title: Segmental progression in PD

Abstract word count: 244

Text word count: 2697

References: 17

Table: 1

Figures: 3

Authors contributions: WMMS: conception and design, acquisition of data, analysis and interpretation of data, drafting of the manuscript. JCC: analysis and interpretation of data, critical revision of the manuscript, statistical expertise. VC: conception and design, acquisition of data, drafting of the manuscript. MBD: acquisition of data, drafting of the manuscript. JLG: analysis and interpretation of data, drafting of the manuscript, statistical expertise. YA: conception and design, critical revision of the manuscript. AH: conception and design, analysis and interpretation of data, drafting of the manuscript.

"The Corresponding Author has the right to grant on behalf of all authors and does grant on behalf of all authors, an exclusive licence (or non exclusive for government employees) on a worldwide basis to the BMJ Publishing Group Ltd and its licencees, to permit this article (if accepted) to be published in JNNP and any other BMJ Group products and to exploit all subsidiary rights, as set out in our licence (http://jnnp.bmjjournals.com//ifora/licence.pdf)".

Acknowledgements: We thank the research nurses and the technicians at the CIC for their professional help in organizing this protocol. We thank Dr Merle Ruberg for critically reading 
this manuscript. M. Schüpbach was supported by grants from the Swiss National Science Foundation and the Swiss Parkinson's Disease Association. 


\section{Abstract}

Objective: To assess the ability of potentially neuroprotective compounds to slow the progression of Parkinson's disease (PD), sensitive rating scales are needed to detect clinically meaningful effects. We evaluated the topographical progression of motor signs in early untreated PD to complement current clinical ratings and enhance the sensitivity to detect disease progression. Methods: Twelve patients referred for diagnostic evaluation of so far untreated de novo PD underwent detailed clinical assessment of motor parkinsonian signs at baseline, 6, and 12 months' follow-up using the Unified Parkinson's Disease Rating Scale, motor part (UPDRS-III), and a newly developed approach of detailed segmental rating taking into account the localization of motor signs in all the major joints and muscle groups in the body. The progression of PD as measured with the UPDRS-III was compared to the segmental ratings. Results: UPDRS-III scores and segmental ratings for rigidity and rest and postural tremor, but not bradykinesia, progressed significantly during the observation period. Progression of normalized segmental ratings for rigidity and tremor was significantly larger than the UPDRS-III ratings over one year. The segmental ratings for rigidity and tremor as well as their combination with the UPDRS-III bradykinesia rating were more sensitive a measure for progression of PD than the UPDRS-III. Conclusions: Taking into account the segmental evolution of parkinsonian signs may be a useful adjunct to UPDRS-III evaluations to measure clinical disease progression of PD. If validated in subsequent, larger cohorts, this may be useful in trials of neuroprotective agents.

Keywords: Parkinson, progression, de novo, rating, pilot study, proof-of-concept 


\section{INTRODUCTION}

The largest unmet need in the current treatment of Parkinson's disease (PD) is a neuroprotective compound that can slow or even halt disease progression. Basic research has identified a wide array of potentially neuroprotective molecules that are effective in in vitro and in animal models of PD, but the clinical testing of such molecules is a formidable challenge because of the hundreds of (de novo) patients required for such trials and the need for lengthy follow-up periods (1). The idea of performing exploratory neuroprotection trials to screen for effective compounds, which may then be tested in larger patient cohorts over prolonged periods of time (2) is attractive, but requires more sensitive tools to assess neuroprotection than those currently available. A more discriminating method for assessing the clinical status of the patients would be helpful in this respect.

The degeneration of dopaminergic neurons in the substantia nigra pars compacta in PD follows a specific spatio-temporal gradient along the rostral, medial, and dorsal axes $(3,4)$. The nigrostriatal neurons along these axes project to specific regions of the striatum, where the body parts - legs (dorsolateral putamen), face (ventromedial putamen) and arms (inbetween the two afore-mentioned areas) - are selectively represented (5). Therefore, the body parts clinically affected would be expected to evolve following an individual spatial gradient along the body over time. This was tested in one retrospective study (6), but no prospective study has been conducted to date in which the somatotopic progression of signs was evaluated.

The Unified Parkinson's Disease Rating Scale, part III (UPDRS-III) (7), the scale most widely used to assess the severity of PD motor signs, has been used as a primary outcome measure in trials assessing neuroprotection. The UPDRS-III provides a good overall estimate of disease severity, most particularly akinesia and bradykinesia (8). Whereas distal and more proximal bradykinesia is differentially assessed with the UPDRS-III, tremor and rigidity are 
rated for each limb as a whole, not taking into account the segmental progression along each arm or leg. The consequence is that identical UPDRS-III scores may be obtained for a severe focal deficit and a widely distributed, but mild impairment.

In this study, we prospectively analyzed the anatomical progression of signs in de novo PD patients, over a period of 12 months, with a newly designed clinical rating approach that evaluates the cardinal PD signs in all the major joints and muscle groups in the body. In this pilot, proof-of-concept approach we suggest that including the segmental progression of tremor and rigidity in the clinical rating of parkinsonian signs may improve the sensitivity of the assessment of disease progression.

\section{Patient And Methods}

\section{Patients}

Twelve de novo PD patients, seven men and five women, who did not require immediate dopaminergic treatment, were prospectively recruited in our outpatient clinic over a period of 14 months. All fulfilled established diagnostic criteria for PD (9), except for their response to levodopa, which was not assessed. After evaluation of their state at inclusion (Month 0), patients were again assessed after 7.5 \pm 1.7 months (M6), and 13.6 \pm 2.6 months (M12). Two patients could not be examined at the intermediate time point. Ten patients had rest tremor at inclusion, the remaining two had a predominantly akineto-rigid form of PD initially but developed mild rest tremor during the study period.

\section{Clinical assessment}

\section{History and examination}

At inclusion (M0), the patients were interviewed for their medical and surgical history, exposure to toxins, current and past medication, family history of neurological disorders, 
educational level, socio-professional status, and their neurological signs, with special emphasis on sleep disorders, hyposmia and dysautonomia, a detailed history of parkinsonian signs and symptoms, with a special emphasis on evolution and modulating factors. Weight, height and vital signs were recorded, and a complete medical and neurological examination was performed by the same examiner throughout the study.

\section{Classical rating scales}

At each visit (M0, M6 and M12), the patients were examined with the UPDRS parts I-III (7), as well as the Hoehn and Yahr (10) and the Schwab and England scales (11).

\section{Segmental evaluation of signs}

The severity of rigidity was rated, with the patient supine and maximally relaxed, on a scale ranging from 0 to 5 : 0 , no rigidity; 1 , no rigidity at rest, but rigidity when the contralateral arm was lifted (Froment's sign) (12); 2, minimal rigidity, detected by slight pressure on the joint, present at rest; 3 , slight rigidity, detected easily by the examiner, but passive movement is not limited, and rigidity is overcome with very little effort; 4, moderate rigidity that

requires some effort to overcome, but passive movement is not limited; 5, pronounced rigidity that requires clear effort to overcome, and passive movement is limited (equivalent to UPDRS rating 3). Rigidity was assessed in the neck, by flexion/extension and rotation, in the trunk by rotation of the patient's shoulders either standing or seated, in the left and right shoulder by both rotation and elevation, in the left and right elbow by flexion/extension and pronation/supination rated separately, in the left and right wrist, hip (rotation), knee and ankle. Rating of rigidity required 5 to 10 minutes. The sum of the ratings provided a rigidity severity score (R-sev). The number of locations where the rigidity score was not 0 , provided a topographical rigidity score (R-top). 
The severity of rest tremor (maximal amplitude observed during the examination) was assessed on the face/lips, the head/neck, the fingers, hands, forearms, upper arms, thighs, calves, and feet, on a five point scale, both relaxed and during a mental effort (repeatedly subtracting 7 from 100; naming the last 5 French presidents backwards): 0, absent; 1, minimal (amplitude $<0.5 \mathrm{~cm}$, i.e. barely visible); 2 , slight (amplitude $<1 \mathrm{~cm}$, i.e. visible but discrete); 3 , moderate (amplitude $>1 \mathrm{~cm}$ ); 4 , severe (amplitude $\geq 5 \mathrm{~cm}$ ). Since rest tremor in the relaxed state varied greatly as a function of the patients' psychological tension during the examination, which was videotaped, only the ratings during a mental effort were analyzed.

Postural tremor, assessed proximally and distally in all four limbs, and action tremor, also assessed on all four limbs, was scored in the same way as rest tremor. Rating of tremor required 5 to 10 minutes. A tremor severity score (T-sev) and a topographical tremor score (T-top) were assigned for rest, postural and action tremor, individually, as described for rigidity.

Akinesia was assessed by repetitive tapping tasks at the following locations bilaterally: all fingers, with the forearm and hand fixed to a surface to limit wrist movement; wrists, with the forearm fixed on a surface; legs, stepping up and down on a $43 \mathrm{~cm}$ high stool; feet, tapping with the sole while the heel rests on the ground, tapping alternately with the heel and the toes, and tapping with the big toe with the foot fixed on a surface. The number of taps per joint recorded over a 30 second period was the outcome measure for each segmental bradykinesia test. A tapping test according to the CAPSIT was performed for each arm during 60 seconds (13). Patients were asked to move as quickly as possible. The experimental setting included a mechanical counter for the repetitive movements.

\section{Composite scores}


A composite tremor and rigidity severity (c-sev) score combined the severity scores for rigidity and rest tremor. A total severity score included the c-sev score and the UPDRS-III bradykinesia ratings (items 23-26, and item 31). A composite topographical tremor and rigidity (c-top) score for rigidity and rest tremor combined the localization scores for rigidity and tremor. These scores were constructed post hoc.

\section{Statitistical analysis}

The main outcome measures were the c-sev score, the c-top score and the total severity score. All outcome measures were analyzed with repeated measures ANOVA to assess progression over time. If the ANOVA yielded a significant result for a given measure, a Tuckey test was performed to assess progression during the first (M0-M6) and second half (M6-M12) of the observation period. A $p \leq 0.05$ was considered significant. However, as multiple tests were performed to explore the segmental progression of bradykinesia, a $\mathrm{p} \leq 0.002$ was considered significant for the measures of bradykinesia.

To compare our new scores with the UPDRS-III in terms of sensitivity for progression, three pair-wise comparisons were performed, between each of the three new scores (c-sev, c-top, and total severity score) and the UPDRS-III. For each comparison, three statistical approaches were used: (1) A repeated measures ANOVA was conducted on the raw data for assessing the interaction between the assessment number (M0, M6, M12) and the scale chosen (UPDRS-III or one of the new scales), that is the lack of parallelism between the score curves. As the patients served as their own controls, a paired design was chosen. (2) The ratings were normalized by calculating a z-score (subtraction of the mean at M0, then division by the SD at M0); then progression as measured with the different scales has been compared by comparing the distributions of differences between M0 and M6 and M0 and M12 using Wilcoxon's 
signed rank test. (3) The ratings were normalized with respect to baseline defined as $100 \%$; then the relative increments were compared using paired Wilcoxon signed rank sum tests. A $p$ value of $\leq 0.05$ was considered significant and all the tests were two-sided. Computations were performed using the SAS V8 statistical package.

Inter-rater agreement was assessed for tremor using videotapes of the assessments. The raters were blinded to all previous ratings given. The inter-rater correlation coefficient $\kappa$ (kappa) was calculated as described elsewhere (14). For inter-rater agreement with identical ratings, $\kappa$ was 0.50 . For inter-rater agreement \pm 1 point, $\kappa$ was 0.91 .

\section{RESULTS}

Mean age at first symptom was $52.2 \pm 8.0$ years, and mean age at inclusion was $53.9 \pm 7.8$ years, with a mean of $20.9 \pm 15.7$ months since the very first putative clinical manifestation of PD. Parkinsonian signs began on the left side in 8 patients, on the right side in 4 . Ten patients were right-handed; two were left-handed and trained at school to be ambidextrous. The initial signs or symptoms of each patient are shown in Figure 1. No patient reported exposure to toxins. Family history for rest tremor and parkinsonism was negative in all patients for first and second degree relatives.

Rigidity was initially found in the upper extremities in half of the patients and in the upper and lower extremities in the other half; at M12, all of the patients had rigidity in the upper and lower extremities. It was bilateral in only 4 patients at M0, but in 11 at M12. Rest tremor was initially observed in the upper and lower extremities of 4 patients, in the upper extremity only in 5, and in the lower extremity only in one. Two patients did not have tremor initially. Rest tremor spread to the upper and lower limbs in 9 patients, but remained restricted to the upper extremities in 3 . It was unilateral at M0 in all 10 patients who had tremor initially, and 
became bilateral in 6 by M12. The two patients who had no tremor initially developed unilateral rest tremor by M12.

Rigidity often progressed faster than tremor. None of the patients developed bilateral tremor before bilateral rigidity and 4 developed bilateral tremor and rigidity simultaneously. Only one patient had unilateral rigidity and tremor for the 12 month period of the study. The segmental evolution of the signs in individual patients is illustrated in Figure 2.

During the course of the study, the Hoehn and Yahr stage, the scores on the Schwab and England and UPDRS II and III scales, as well as the subscores on the UPDRS-III (tremor, rigidity, and bradykinesia score) worsened significantly (Table 1). Segmental rigidity, rest and postural tremor scores also worsened from M0 to M12 (Table 1). The UPDRS I, the UPDRSIII axial score and the segmental action tremor and bradykinesia scores did not change significantly during the study period (Table 1). As the segmental bradykinesia ratings were insensitive to change over time and because we wanted to include the aspects of bradykinesia in a global score, the UPDRS-III bradykinesia score was used (rather than the segmental bradykinesia ratings) to build the total severity score.

According to the UPDRS-III evaluation, parkinsonian signs progressed $192 \pm 47 \%$ (from $13.42 \pm 5.93$ to $24.25 \pm 7.03$ ) between M0 and M12, but $259 \pm 75 \%$ when the c-sev score was used, $248 \pm 95 \%$ when the c-top score was used, and $227 \pm 52 \%$ when the total severity score was used (Figure 3). The slope of progression of the UPDRS-III score was less steep than the slope of progression of the $\mathrm{c}-\mathrm{sev}$ score $(\mathrm{p}=0.0022)$ and the total severity score $(\mathrm{p}=0.0001)$, but not the c-top score. However, all three composite scores were more sensitive than the UPDRS-III to detect clinical progression of PD defined as the normalized difference of follow up ratings compared to baseline after 12 months (p-values are given for normalization with a $\mathrm{z}$-score and for normalization with percentage, respectively: for the c-sev score: $\mathrm{p}=0.02 / 0.005$; for the c-top score $\mathrm{p}=0.001 / 0.02$; for the total severity score $\mathrm{p}=0.03 / 0.02$ ). After 6 months, 
there was no significant difference in terms of detection of progression of PD between the UPDRS-III and the composite scores.

\section{DisCUSSION}

This pilot study demonstrates that a segmental examination of tremor and rigidity in de novo PD patients, combined with the bradykinesia scores from the UPDRS III, is more sensitive for detecting changes in disease severity over one year than the UPDRS-III.

In our study, rigidity evolved faster but in a similar distribution pattern as tremor. This may be due to the fact that rigidity is more easily detected than tremor, or maybe just more apparent on examination. Increasing rigidity may mask rest tremor (15). A recent multitracer positron emission tomographic study of patients with monosymptomatic resting tremor compared to early PD patients displaying all three cardinal signs suggested that rigidity and tremor correlated with a presynaptic dopaminergic deficit along an anteroposterior putaminal gradient (16). This supports our observation that the segmental evolution of rigidity and tremor is similar and reflects a common nigrostriatal dopaminergic deficiency, at least in early disease stages.

Akinesia/bradykinesia scores obtained in the segmental repetitive movement tests unexpectedly did not change over the 12 month study period, in contrast to the UPDRS-III items that assess akinesia/bradykinesia. The difficulty inherent in the tests may be responsible, in that they required the active cooperation of the patient. Indeed, the voluntary effort most of our patients made during these quantitative tapping tests - to prove to themselves that PD had not measurably progressed - might have compensated for potentially worsened motor impairments, especially at this early stage of the disease. The quantification of the UPDRS bradykinesia ratings is not obvious for the patients during the exam, and there may have been less incentive to outperform the baseline rating than in the tapping tasks where 
performance is quantified on a counter visible to the patient during the effort. It was previously suggested that attention can compensate to some degree for the basal ganglia deficit responsible for akinesia/bradykinesia (17). However, these authors also proposed that the engagement of compensatory processes may lead to reduced performance in other tasks, which would become apparent when running two tasks simultaneously (17). In accordance with this, we observed in many patients an increase of tremor in the limbs not involved in tapping during these tasks. Thus, we do not exclude that akinesia/bradykinesia can be unmasked and possibly amplified in a segmental evaluation (compared to UPDRS-III items) if a further simultaneous task were added. Although we applied established diagnostic criteria for PD, the age of onset in our patients was relatively young, and levodopa response could not be evaluated in all patients. However, the aim of this pilot study was to clinically assess parkinsonian signs, and not to ascertain a diagnosis of PD.

In conclusion, we believe that our segmental rating approach will usefully complement the UPDRS-III akinesia/bradykinesia section and increase the sensitivity of clinical evaluations. The goal of this preliminary pilot study was to provide a proof of concept. Although the detection of clinical progression was significantly enhanced using a segmental tremor and rigidity rating, even in a small group of patients, further validation of the new scores in a larger cohort is needed. Yet, within small exploratory neuroprotection trials where one investigator studies all patients, this method may be a worthwhile adjunct to UPDRS-III testing in a foreseeable future. 


\section{REFERENCES}

1 Clarke CE. Neuroprotection and pharmacotherapy for motor symptoms in Parkinson's disease. Lancet Neurol 2004;3:466-474.

2 Choi DW. Exploratory clinical testing of neuroscience drugs. Nat Neurosci 2002;5 Suppl:10231025.

3 Damier P, Hirsch EC, Agid Y, Graybiel AM. The substantia nigra of the human brain. I. Nigrosomes and the nigral matrix, a compartmental organization based on calbindin $\mathrm{D}(28 \mathrm{~K})$ immunohistochemistry. Brain 1999;122:1421-1436.

4 Damier P, Hirsch EC, Agid Y, Graybiel AM. The substantia nigra of the human brain. II. Patterns of loss of dopamine-containing neurons in Parkinson's disease. Brain 1999;122:1437-1448.

5 Brown LL, Sharp FR. Metabolic mapping of rat striatum: somatotopic organization of sensorimotor activity. Brain Res 1995;686:207-222.

6 Dickson JM, Grunewald RA. Somatic symptom progression in idiopathic Parkinson's disease. Parkinsonism Relat Disord 2004;10:487-492.

7 Fahn S, Elton RL, UPDRS Development Committee. Unified Parkinson's disease rating scale. In: Fahn S, Marsden CD, Calne D, Goldstein M, editors. Recent developments in Parkinson's disease. Vol 2. Florham Park, NY:MacMillan Healthcare Information; 1987:153-163.

8 Ramaker C, Marinus J, Stiggelbout AM, Van Hilten BJ. Systematic evaluation of rating scales for impairment and disability in Parkinson's disease. Mov Disord 2002;17:867-876.

9 Hughes AJ, Daniel SE, Ben-Shlomo Y, Lees AJ. The accuracy of diagnosis of parkinsonian syndromes in a specialist movement disorder service. Brain 2002;125:861-870.

10 Hoehn MM, Yahr MD. Parkinsonism onset, progression and mortality. Neurology 1967;17:427442.

11 Schwab JF, England AC. Projection technique for evaluating surgery in Parkinson's disease. In: Gillingham FJ, Donaldson ML, editors. Third symposium on Parkinson's disease. Edinburgh, 
Scotland: E \& S Livingstone Ltd; 1969:152-157.

12 Broussolle E, Krack P, Thobois S, Xie-Brustolin J, Pollak P, Goetz CG. Contribution of Jules Froment to the study of parkinsonian rigidity. Mov Disord 2007;22:909-914.

13 Defer GL, Widner H, Marié RM, Rémy P, Levivier M. Core assessment program for surgical interventional therapies in Parkinson's disease (CAPSIT-PD). Mov Disord 1999;14:572-584.

14 Altman DG. Practical statistics for medial research. Chapman \& Hall/CRC, London 1999:403-409.

15 Winogrodzka A, Wagenaar RC, Bergmans $\mathrm{P}$ et al. Rigidity decreases resting tremor intensity in Parkinson's disease: A [(123)I]beta-CIT SPECT study in early, nonmedicated patients. Mov Disord 2001;16:1033-1040.

16 Ghaemi M, Raethjen J, Hilker R et al. Monosymptomatic resting tremor and Parkinson's disease: a multitracer positron emission tomographic study. Mov Disord 2002;17:782-788.

17 Berardelli A, Rothwell JC, Thompson PD, Hallett M. Pathophysiology of bradykinesia in Parkinson's disease. Brain 2001;124:2131-2146. 


\section{FIGURE LEGENDS}

(The underlined words can serve as figure titles)

Figure 1: Initial symptoms of Parkinson's disease in the 12 patients studied. T: rest tremor. R: rigidity. $\mathrm{P}$ : pain preceding rigidity. B: bradykinesia. M: micrographia.

Figure 2: Illustration of the topographical progression of rest tremor and rigidity in 6 exemplary patients. The data of one patient are grouped in one box. The upper row within a box refers to rest tremor (red/orange); the lower row within a box refers to rigidity (blue). Within one box, progression is illustrated from M0 (left) over M6 (middle) to M12 (right). Symptom severity is indicated by the number of dots according to the 6 point rating scale. Red dots stand for rest tremor under relaxed condition; orange dots indicate the worsening of rest tremor during a mental task. R: right. L: left.

Figure 3: Progression of clinical signs of PD as assessed with the three composite scores and the UPDRS-III. Baseline values are set at $100 \%$. T: rest tremor. R: rigidity. B: bradykinesia 


\section{Initial symptom in the 12 patients}
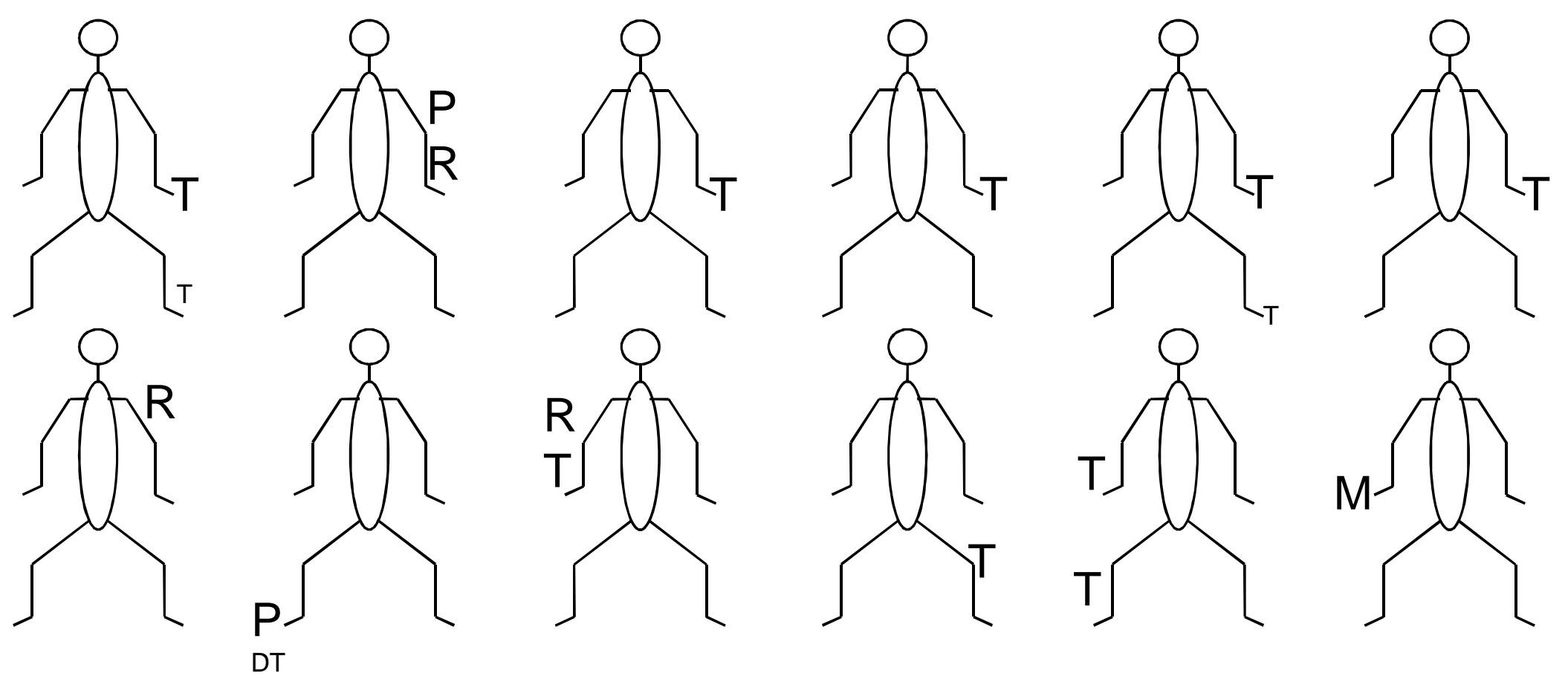

$\mathrm{R}$

T tremor; $\mathrm{R}$ rigidity; $\mathrm{P}$ pain, DT dystonia, $\mathrm{M}$ micrographia 
Fig. 2
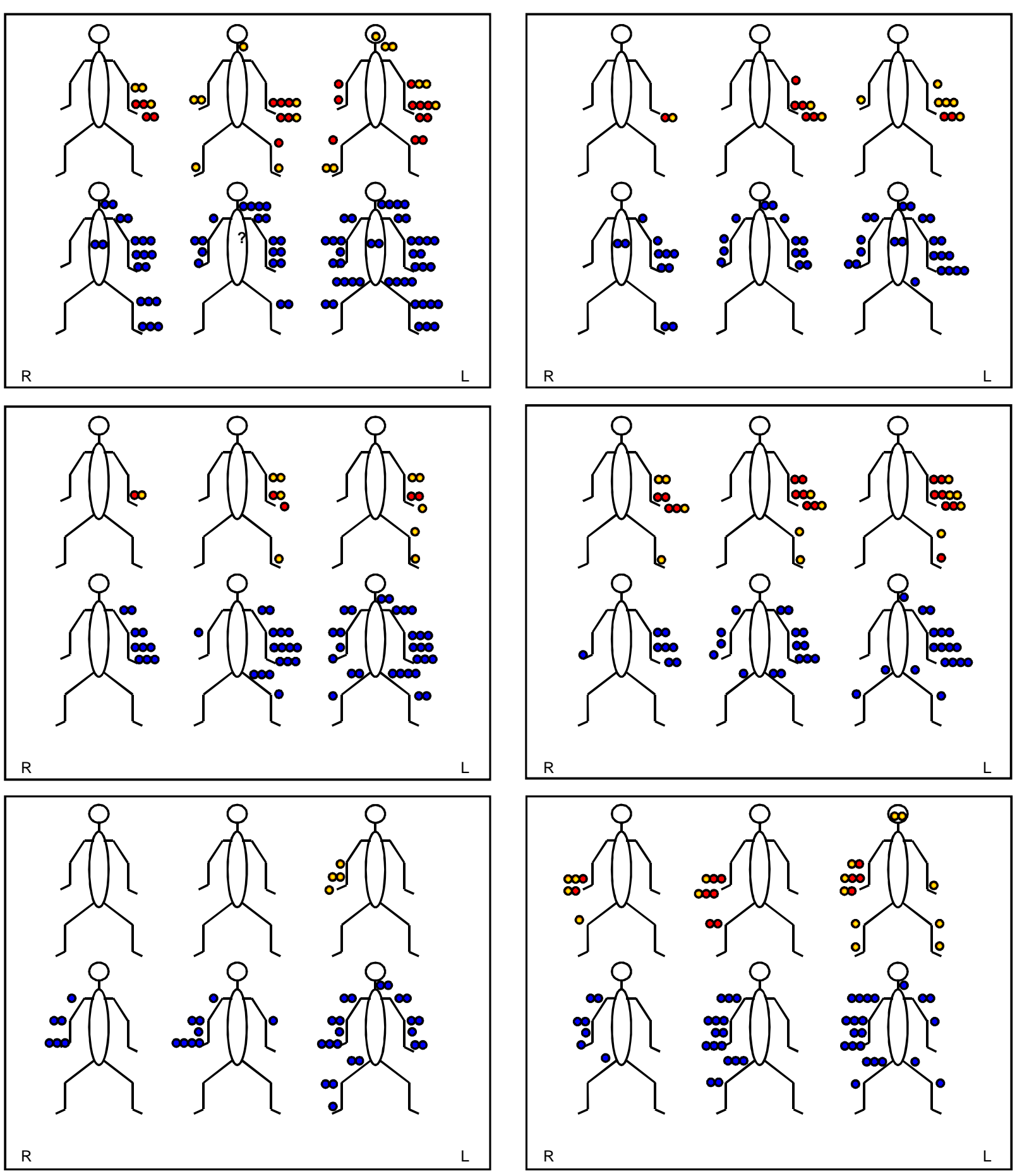


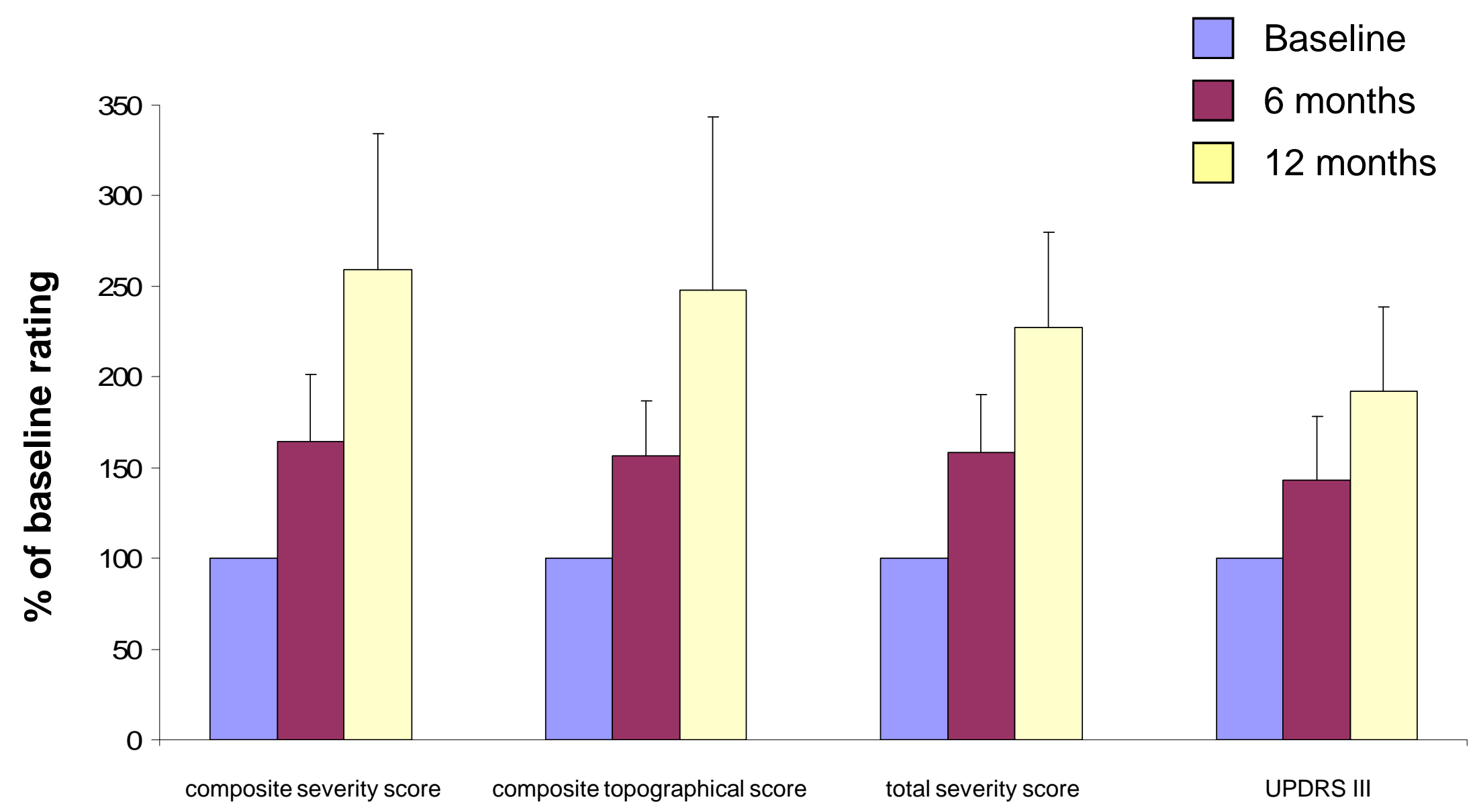

